\title{
BMJ Open Status of hospital-based blood transfusion services in low-income and middle-income countries: a cross- sectional international survey
}

\author{
Linda S Barnes (D , , ${ }^{1,2}$ Jean Stanley, ${ }^{3}$ Evan M Bloch, ${ }^{4}$ Monica B Pagano, ${ }^{5}$ \\ Tina S Ipe, ${ }^{6}$ Quentin Eichbaum, ${ }^{7}$ Silvano Wendel, ${ }^{8}$ Alexander Indrikovs, ${ }^{9}$ Wei Cai, ${ }^{10}$ \\ Meghan Delaney, ${ }^{11}$ On behalf of the AABB Global Transfusion Forum
}

To cite: Barnes LS, Stanley J, Bloch EM, et al. Status of hospital-based blood transfusion services in lowincome and middle-income countries: a cross-sectional international survey. BMJ Open 2022;12:e055017. doi:10.1136/ bmjopen-2021-055017

- Prepublication history and additional supplemental material for this paper are available online. To view these files, please visit the journal online (http://dx.doi.org/10.1136/ bmjopen-2021-055017).

Received 07 July 2021 Accepted 14 January 2022

Check for updates

(C) Author(s) (or their employer(s)) 2022. Re-use permitted under CC BY-NC. No commercial re-use. See rights and permissions. Published by BMJ.

For numbered affiliations see end of article.

Correspondence to

Dr Linda S Barnes;

Ibarne23@gmail.com

\section{ABSTRACT}

Objectives Blood transfusion is life-saving for patients experiencing acute blood loss and severe anaemia. In lowincome and middle-income countries (LMICs), low blood donation rates and unavailability of whole blood and blood components (blood products) impairs timely blood transfusion. To fulfil patient-specific blood orders, a hospital blood transfusion service (HBTS) receives orders from a prescriber for blood transfusion, tests and prepares blood products for the patient. This study sought to describe the current state of LMIC HBTS.

Design A cross-sectional survey explored LMIC HBTS access to blood products, testing methods, policies and structure. Surveys were administered in English, Spanish, French and Russian, followed by a mixed-methods analysis.

Setting HBTS within LMICs.

Participants From among 124 public and private facilities invited to participate, we received $71(57 \%)$ responses. Of these responses, 50 HBTS from 27 LMICs performed on-site blood transfusions.

Results Most LMIC HBTS perform blood collection to generate blood products for their patients (36/47, $77 \%$ ); few relied exclusively on an external supply of blood products (11/47, 23\%). The primary reason for blood transfusion was adult anaemia for non-malignant conditions (17/112, 15\%). Testing methods varied by gross national income per capita. Blood transfusion delays to patients were common (17/30, $57 \%$ ) attributed to inadequate blood inventories $(13 / 29,45 \%)$. Other barriers included lack of regular clinician education about transfusion $(8 / 29,28 \%)$ and sustainable financial models for the HBTS (4/29, $14 \%)$.

Conclusion This survey describes the status of HBTS in diverse LMICs, illustrating that the availability of blood products remains a principal problem, requiring HBTS to generate its own facility's blood supply. Currently, blood shortages are not reported as a patient-specific adverse event making systematic tracking of delays in transfusion difficult. These findings highlight areas for further exploration related to the lack of available blood inventories for transfusions at HBTS in LMICs.

\section{INTRODUCTION}

In health systems worldwide, blood transfusion is crucial for patients experiencing
Strengths and limitations of this study

- This is one of the largest compilations of hospitalbased blood transfusion capabilities and challenges in diverse low-income and middle-income countries (LMICs).

- The survey provides information on hospital blood transfusion services regarding the blood supply, testing capabilities, education and structure in a part of hospital operations where little is published.

- The purposive sampling plan consisted of known transfusion medicine associates working in LMIC blood transfusion services, improving credibility by accessing individuals familiar with the subject matter and setting.

- Despite ample participation in multiple languages, selection and information bias present limitations to representativeness, generalisability and transferability of the findings to other settings.

acute blood loss or severe anaemia. ${ }^{1}$ Globally, whole blood and blood components (blood products) are essential medicines necessary for blood transfusion, yet shortages are a well-described problem, particularly in low-income and middle-income countries (LMICs) ${ }^{2-4}$ Lack of timely blood transfusion contributes to poor patient outcomes, particularly in women during pregnancy and childbirth and children under 5 years old suffering from severe anaemia. ${ }^{56}$

The gaps contributing to blood shortages for transfusion in LMIC are multifactorial, including low public participation in voluntary non-remunerated blood donation (VNRBD), blood donor suitability, skilled workforce shortages, access to educational programmes, infrastructure limitations and sustainable financial models. ${ }^{7-9}$ Published accounts address these limitations on the national level, including the establishment 
of centralised blood transfusion services and blood safety policies to reduce blood-borne pathogens. ${ }^{2}{ }^{20-13}$ Although the hospital-based transfusion service (HBTS) is a critical part of the safe delivery of transfusion, the literature provides scant information on the provision of blood transfusion at the hospital level within LMIC.

In LMIC, the characteristics of who donates blood and how blood products are provisioned for blood transfusion are variable. ${ }^{14}$ Blood services range from national collection/ supplier programmes to localised hospital-level services, driven by economic considerations, political appetite and health system complexity. ${ }^{15-17}$ Within a hospital, the HBTS is a clinical laboratory (sometimes called a 'blood bank') that prepares, stores and dispenses blood products for patient blood transfusion within the facility. ${ }^{18}$ Notably, the HBTS is responsible for selecting appropriate blood products for the patient based on their ABO and Rh blood type and compatibility requirements (pre-transfusion testing). Therefore, the HBTS serves a pharmacy role by preparing the doctor's prescription to match the patient's blood type and ensure a safe transfusion event. To do this correctly, the HBTS must have testing capabilities, knowledgeable staff and an ample supply of blood products on hand, particularly to provide for emergency transfusions when a patient has life-threatening haemorrhage. Data about capabilities within LMIC HBTS are lacking, including the source of the blood supply, the pretransfusion testing methods used, clinical transfusion practices, and post-transfusion surveillance (eg, reporting of adverse events). ${ }^{819}{ }^{20}$ Understanding the diverse abilities and needs could highlight areas for improvement and protect against the lack of blood transfusion leading to preventable outcomes. ${ }^{5619}$ This study sought to describe the current state of HBTS in LMICs, including the facility and blood supply characteristics, leadership oversight, testing capabilities, patient care factors and challenges in settings that generally have few resources with the purpose of identifying opportunities for improvement and further investigation. ${ }^{10} 1921$

\section{METHODS}

A cross-sectional survey was designed to acquire information about current practices, processes, and structure in LMIC HBTS. Research questions were formulated through an iterative process involving several researchers experienced in LMIC blood transfusion and a literature review of the challenges or gaps observed in blood provision. The survey focused on the blood supply characteristics, organisation, pretransfusion testing methods and challenges experienced at the hospital level. The survey (54 questions, online supplemental file) was developed in English then translated into French, Spanish, and Russian. To optimise participation, the survey met accessibility standards on various devices (eg, smartphone, desktop, tablet) and comprehension level (Flesch-Kincaid). Translations were reviewed by native speakers knowledgeable in transfusion medicine to assure accuracy. The survey sought information about current practices, procedures, and structure of LMIC HBTS through multiple-choice, best-choice, Likert-type responses, categorical selections, open-ended questions and quantification. The survey was piloted by members within the research team who did not build the questionnaire to improve readability, reduce length, refine data capture and increase ease of use.

Survey participants were identified by the research team. The participants were from LMIC countries and differentiated by World Bank Atlas categories. ${ }^{22}$ Because there is no global registry of HBTS, potential respondents were identified by the manuscript authors as verified transfusion medicine contacts in LMICs. The names of potential participants were compiled into a distribution list comprising 124 invitees. Data collection occurred between 24 February 2020 and 1 April 2020, using email distribution within Qualtrics XM, Version 2020 (Qualtrics, Provo, Utah, USA). A copy of the AABB Fundamentals Standards for Blood Banks and Transfusion services was offered as a nominal incentive to all respondents. ${ }^{18}$ Responses from sites that did not perform blood transfusion at the facility to assure reporting of direct experience were excluded. Data were tabulated and analysed using Excel (Microsoft, Seattle, Washington, USA). The denominator was modified if the question was not answered by all respondents. Statistics used the Fisher's exact test. Descriptive data displays were generated in the form of tables and figures. Qualitative methods, using thematic analysis constructed from free-text comments received from respondents, were applied to an explanatory framework to generate recommendations.

\section{Patient and public involvement}

There was no patient or public involvement in the study.

\section{RESULTS}

A total of 71 complete responses were received for a $57 \%$ response rate. Excluding responses from facilities that did not perform blood transfusion, 50 responses were from 27 different LMIC countries: 4 low (LIC), 11 lowmiddle (LMC), 12 upper-middle (UMC) income countries (table 1). The response languages consisted of 33 English (66\%), 9 Spanish (18\%), 4 French (8\%) and 4 Russian $(8 \%)$. Of the 31 respondents describing their role, $65 \%$ identified as medical directors $(20), 19 \%$ as lab manager/director (6) and $16 \%$ as supervisor (5).

\section{Facility and blood supply characteristics}

There were 18 public hospitals (36\%), 9 private hospitals $(18 \%), 3$ centralised transfusion services serving more than one hospital $(6 \%)$, and $20(40 \%)$ were not categorised. Of the 31 respondents who described their setting, $28(90 \%)$ were situated in a densely populated urban setting and $22(71 \%)$ identified as teaching facilities. Across 33 HBTS reporting their transfusion activity, a median of 6000 units (4-121 987) were transfused in 2019. The respondents also performed the following services: outpatient transfusion to clinics $(21,31 \%)$, 
Table 1 Facility and blood supply characteristics

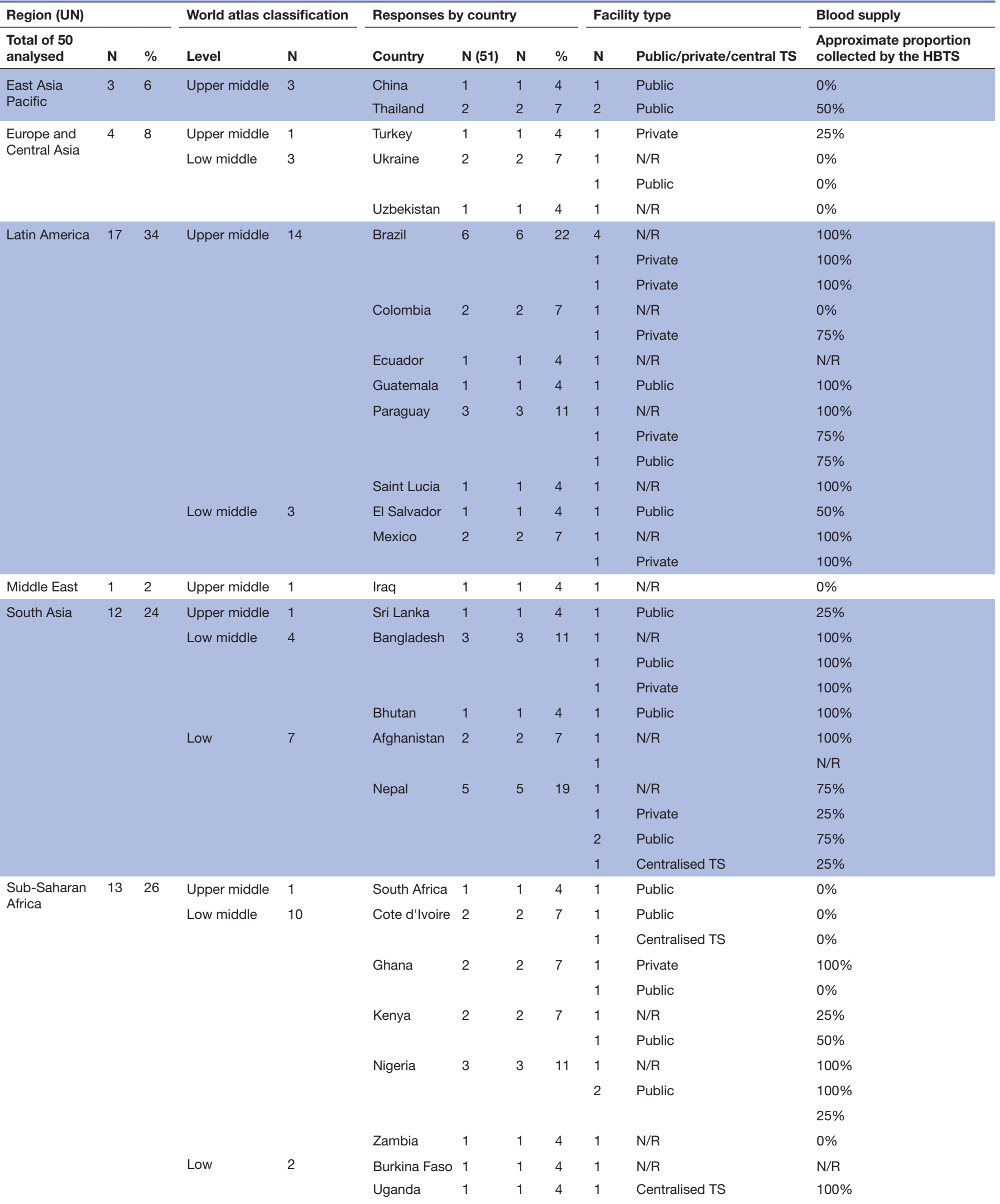

Regions align with the United Nations (UN) designations and classification is by World Bank Category (2019). ${ }^{22}$ A centralised transfusion service supports more than one hospital. Approximate proportion of blood supply collected by the HBTS facility refers to self-reported proportion of blood collected for transfusion internally for use within the hospital (selfcollection).

HBTS, hospital blood transfusion service; NR, not reported; TS, Transfusion Service. 
transfusion testing for other facilities $(18,27 \%)$ and evaluation of transfusion reactions $(28,42 \%)$.

Unlike most high-income countries, $77 \%$ of the HBTS (36 of 47) reported performing blood collections to meet their patients' blood transfusion needs. Of these, 15 HBTS (32\%) described being solely dependent on blood they collected to transfuse their patients (internal hospital-based blood collection). Another 21 HBTS (45\%) performed both internal blood collection and received varying levels of blood supplied from an outside agency (external blood supplier). Only 11 HBTS (23\%) had blood provisioned entirely by an external blood agency. For the HBTS that collected blood, the most common methods of donor recruitment included an open call for blood donation (donors self-identify) (25, $54 \%$ ); hospital policy requirements for patients or family members to supply or replace blood transfused (family or replacement donations) $(15,33 \%)$ or non-monetary incentives $(6,13 \%)$. There were no reports of monetary remuneration to blood donors.

\section{Oversight and leadership}

The leadership of HBTS was varied (table 2). When led by a physician, the medical specialisations involved included haematology/haemotherapy, transfusion medicine and clinical pathology/pathology. Hospital transfusion committees (HTCs) that oversee blood transfusion policies, procedures and guidelines were present in most facilities. The composition of the HTC involved medical doctors, nurses, laboratory staff and administrators, with lesser participation from other stakeholders. Of the 24 HBTS reporting HTC meeting frequency, most met two to four times in the prior year (13, $54 \%)$, followed by more than 10 times $(4,17 \%)$; only two did not meet within the preceding year $(2,8 \%)$. Written blood transfusion policies were present in most reporting facilities. Blood transfusion policies were created at the organisational $(23,44 \%)$, national $(21,40 \%)$ levels or by the department or provider $(8,16 \%)$. The written policies emphasised transfusion reactions, informed consent and the appropriate use of blood.

\section{Pretransfusion testing methods}

The selection of blood for transfusion requires the HBTS to perform patient blood typing, and blood donor-to-patient cross-match testing using immunohaematology (usually serological) laboratory assays to ensure the blood is compatible. Respondents (31) used several different laboratory testing methods (figure 1). The primary methods for blood typing (per the $\mathrm{ABO}$ system: $\mathrm{O}, \mathrm{A}, \mathrm{B}$, and $\mathrm{AB}$ blood groups and Rhesus factor, also known as $\mathrm{ABO} / \mathrm{Rh}$ ), red blood cell (RBC) antibody detection, RBC antibody identification, and compatibility (donor and patient) testing diverged by World Bank classification level. More simplistic testing (tile or slide agglutination) was done in HBTS in LICs, while more reproducible and standardised testing methods (gel or solid phase) used in LMC or UMC. Two facilities of the 31 did not typically perform antibody identification. Of 33 respondents describing pretransfusion compatibility testing of the patient for whole blood and RBC transfusion, most performed
Table 2 Characteristics of HBTS leadership and blood transfusion oversight

\begin{tabular}{|c|c|}
\hline Hospital-level structures & $N=31$ \\
\hline Transfusion service leadership composition & $31(100 \%)$ \\
\hline Medical director & $12(39 \%)$ \\
\hline Manager/director/supervisor & $10(32 \%)$ \\
\hline Other specialist (SBB, pharmacist) & $9(29 \%)$ \\
\hline $\begin{array}{l}\text { Transfusion committee is present and } \\
\text { composition of membership }\end{array}$ & $24(77 \%)$ \\
\hline Medical directors & $24(100 \%)$ \\
\hline Nurses & $20(83 \%)$ \\
\hline TS laboratory staff & $18(75 \%)$ \\
\hline Administrators & $16(67 \%)$ \\
\hline External blood supplier & $8(33 \%)$ \\
\hline $\begin{array}{l}\text { Other (social worker, ethicist, blood donor, } \\
\text { patient) }\end{array}$ & $4(16 \%)$ \\
\hline Ministry of health & $3(13 \%)$ \\
\hline Community members & $3(13 \%)$ \\
\hline Written blood transfusion policies and topics & 29 (94\%) \\
\hline Transfusion reaction notification to the TS & $26(90 \%)$ \\
\hline Informed consent for blood transfusion & $24(83 \%)$ \\
\hline Clinical use of blood & $24(83 \%)$ \\
\hline $\begin{array}{l}\text { Pretransfusion and post-transfusion patient } \\
\text { care }\end{array}$ & $23(79 \%)$ \\
\hline Monitoring of blood transfusion practices & $23(79 \%)$ \\
\hline Massive blood loss and management & $21(72 \%)$ \\
\hline Patient blood management & $21(72 \%)$ \\
\hline Preoperative or preintervention patient care & $19(66 \%)$ \\
\hline Postoperative or postintervention patient care & $15(52 \%)$ \\
\hline Blood product wastage & 15 (52\%) \\
\hline None of the above & $0(0 \%)$ \\
\hline
\end{tabular}

HBTS leadership characterised the background of the individual responsible for leading the service. Transfusion committee composition shows the diversity of participation from the responses; totals exceed $100 \%$ due to multiple selections. ${ }^{45}$ Blood Transfusion Policies illustrate the proportion of respondents having written policies advanced by professional societies. ${ }^{1841}$

HBTS, hospital blood transfusion service; SBB, Specialist in Blood Banking; TS, Transfusion Service.

testing before transfusion $(29,88 \%)$; fewer in certain circumstances $(2,6 \%)$ or not at all $(2,6 \%)$.

\section{Transfusion practice}

The primary reason for blood transfusion was adult anaemia not related to malignancy (figure 2). Emergency transfusion procedures were present in most facilities (27 of $30,90 \%)$; used for trauma $(22,37 \%)$, obstetrical haemorrhage $(19,32 \%)$ and surgery $(18,31 \%)$.

When a patient experienced an adverse reaction from a blood transfusion, most HBTS (29 of 31, 94\%) required clinical staff to report it to the lab; only two HBTS did not require mandatory reporting. Of the types of adverse events requiring reporting, under-transfusion or delayed blood transfusion seldom necessitated formal 


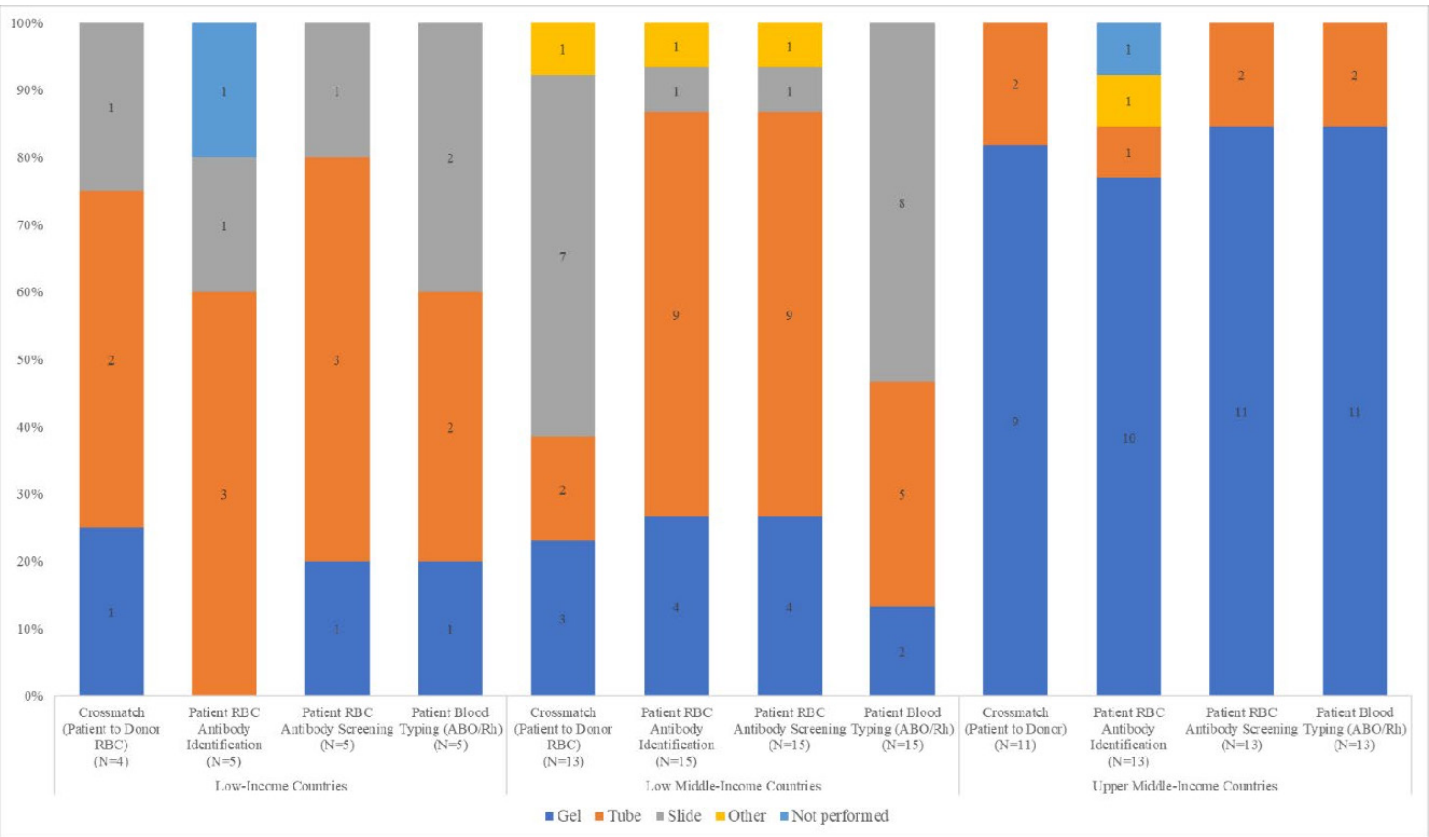

Figure 1 All testing uses agglutination. Blood typing ( $A B O / R h$ using the $A B O$ system: $O, A, B$, and $A B$ and Rhesus [Rh] factor) determines the major blood type for red blood cell (RBC) antigens $A, B$ and rhesus (Rh) factor. $R B C$ antibody screening is a serological test for detection of antibodies directed against RBC antigens other than A and B antigens. RBC antibody identification finds the specific RBC antibody or antibodies present to inform clinical significance. Cross-matching or compatibility testing consists of the cumulative examination of donor and patient major ABO incompatibilities and clinically significant antibodies. Slide or Tile agglutination testing will only detect incompatibility caused by lgM, while tube, gel or solid phase may also detect incompatibility caused by IgG antibodies. The 'other' category included computer cross-match or solid phase capture. Country classification based on world bank atlas method categories (2019). ${ }^{22}$

communication (6 of 231 multi-selection events, 2.6\%). Many HBTS (21 of 32, 66\%) had to report adverse transfusion events to external authorities, such as national haemovigilance or an external regulatory body, however, undertransfusion (blood ordered but not available for the patient) hardly ever required external reporting ( 5 of 153 multiselected events, $3 \%$ ), despite being a common occurrence.

\section{Challenges and barriers}

Nearly 57\% (17 of 30) of HBTS respondents reported that blood was not available within 24 hours, despite an order for blood transfusion placed by a provider. Of the 28 explanations, the delays were due to inadequate blood supplies $(15,54 \%)$; specially matched blood not available $(8,29 \%)$; or other $(5,22 \%)$. The source of the blood

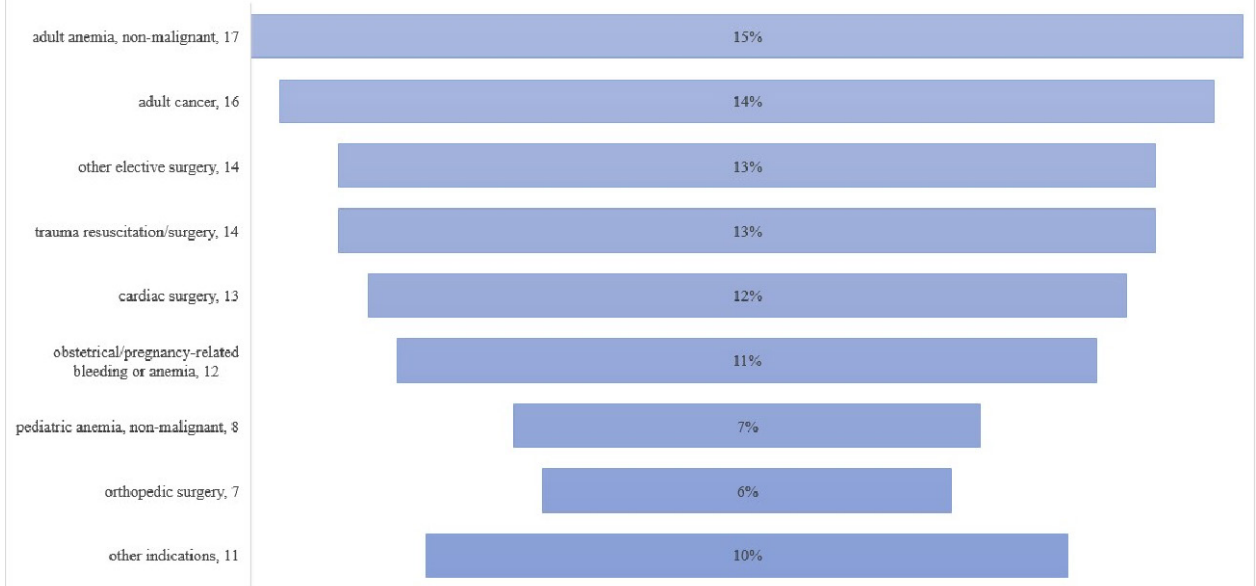

Figure 2 Adult anaemia, non-malignant, includes chronic loss or decreased production of erythrocytes (RBCs) associated with pathophysiological conditions other than cancer. Adult cancer and associated myelosuppressive treatments may decrease RBC production, leading to anaemia, thrombocytopaenia (low platelets) or leucopaenia (low white blood cells). Surgery patients (elective, trauma resuscitation and cardiac, orthopaedic, obstetrical) may require blood transfusion related to acute blood loss, however, underlying anaemia may hasten the need for blood transfusion. RBC, red blood cell. 


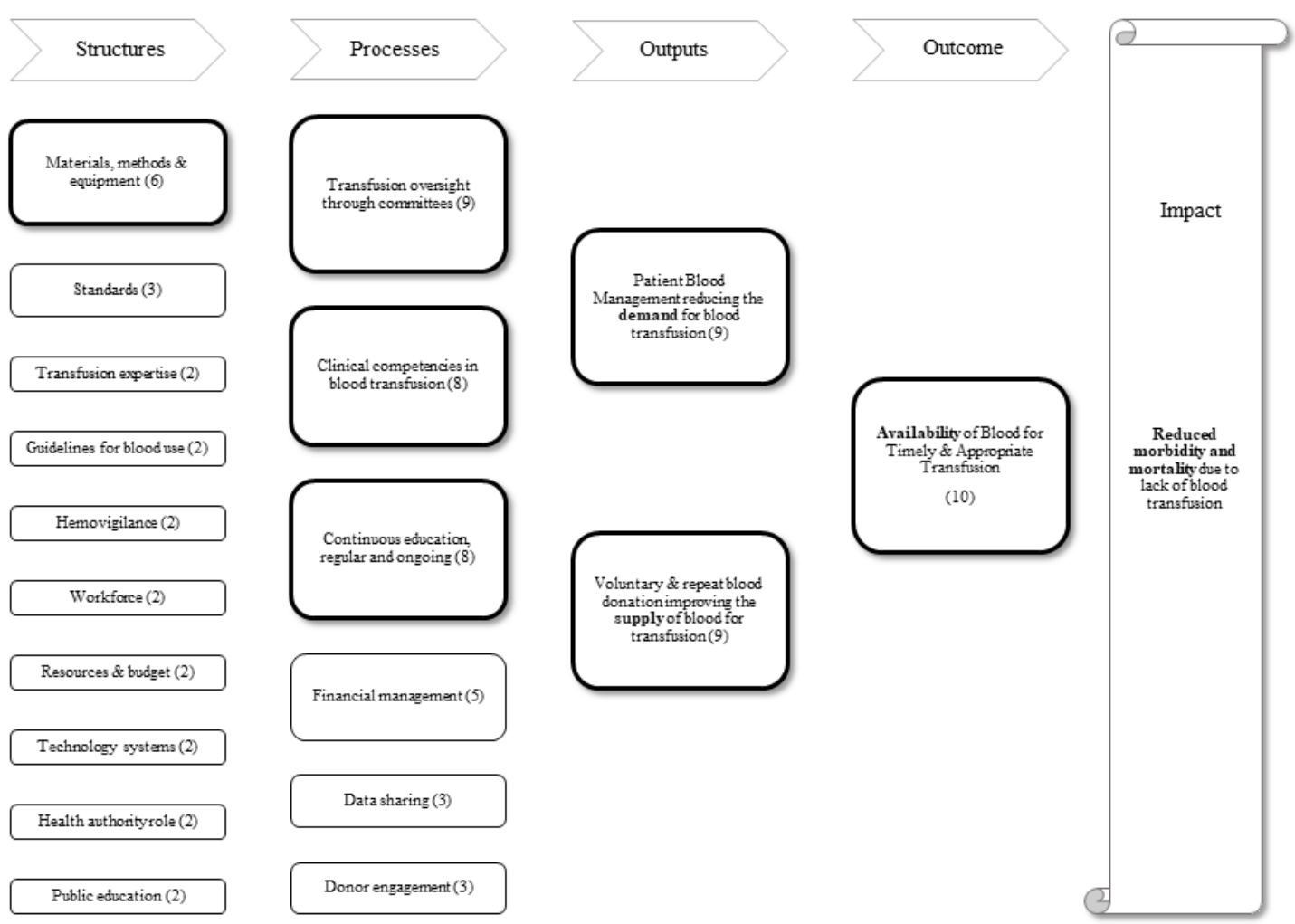

Figure 3 Themes were distilled qualitatively from free-text comments and multiselect options describing the barriers and facilitators encountered by LMIC HBTS for timely and appropriate blood transfusion. The size of the box indicates the relative frequency of mention in responses, the bolded boxes illustrate dominant themes. HBTS, hospital blood transfusion service; LMIC, low-income and middle-income country.

supply was not an independent explanation of blood supply shortages (internal vs external blood supplies [ $\mathrm{p}=0.33$, or comparisons to a hybrid supply versus sole source $[\mathrm{p}=0.46])$, suggesting that other factors, such as overall low VNRBD blood donation rates, were underlying causes.

The availability of the blood supply was the dominant barrier to blood transfusion across the 29 HBTS respondents describing challenges $(13,45 \%)$. Clinician education was the second-most reported factor hindering good transfusion practice $(8,28 \%)$. Educational courses on blood transfusion occurred regularly $(11,37 \%)$; intermittently $(16,53 \%)$ or not at all $(3,10 \%)$. Lack of awareness to report adverse transfusion events emerged as the most significant reason for failures to report suspected transfusion reactions $(19,36 \%)$. Additional barriers included sustainable financial models $(4,14 \%)$ or others (patient education, workforce sufficiency and patient access) (4, $14 \%)$.

Respondents provided recommendations about how to improve the safety and availability of blood transfusions. The responses included themes of (1) increasing the supply of blood through more VNRBD and repeat blood donations and (2) reducing the demand for blood through evidence-based transfusion practices and patient blood management (PBM). These challenges were mapped to a framework describing the steps and resources needed to improve the availability of blood for appropriate transfusion (figure 3). Respondent suggestions included processes to improve HTC oversight, clinical competencies and broad measures to encourage VNRBD. Structural factors emphasised necessary materials, methods and equipment, among other recommendations. Information technology, data sharing and the use of databases emerged as important facilitators to sharing blood resources between HBTS facilities and cultivating repeat VNRBD.

\section{DISCUSSION}

This is the largest survey study assessing the capabilities and blood availability in LMIC hospital-based transfusion services. Much of the published literature about blood transfusion in LMICs focuses on the safety of the blood supply and the risk of transfusion-transmitted diseases. ${ }^{249}$ While these topics are critically important, hospital-based transfusion practices and resources at the local level are essential determinants impacting patient care and outcomes. ${ }^{18} 2123$ The HBTS provides a vital service to ensure enough blood for patients at the facility and that the blood is used safely and efficiently.

Our study substantiated difficulties ensuring adequate blood products for patients at the hospital level. ${ }^{516} 2124$ Respondents described that insufficient blood inventories contributed to delays in treatment for patients. Shortages of blood for timely transfusion were reported across 
all blood supply models, including hospital-based blood collection, blood provisioned from an external supplier, or a combination of both designs. Blood shortages impacting patient care were found across the range of low-income to upper-middle income categories. In highincome countries, the HBTS usually obtains their blood supply from accredited external blood collection agencies (nationalised or not-for-profit organisations) using standardised blood donation programmes, and sensitive and specific laboratory tests to prevent transfusiontransmitted diseases. ${ }^{25}{ }^{26}$ In LMIC, the HBTS must collect blood when there is no such external blood supplier, or the external supplier cannot provide sufficient blood to meet clinical demand. ${ }^{9126}$ However, the contribution of HBTS blood collection activities in this context is poorly documented in the literature.

In our survey, $35 \%$ of the facilities has a policy of family or replacement donation when blood is used from the HBTS. In LMICs, replacement blood donation models rely primarily on patient families and self-organised networks to replace the blood needed by the patient. Relying on family replacement donation (FRD) presents several challenges, such as the potential higher risk of transfusion-transmitted infections, artificial shortages created by reserving blood products for family members or friends, and wastage of unused reserved blood products. ${ }^{1127}$ FRD does not provide a robust blood inventory capable of supporting timely emergency transfusions or fulfilling a robust surgical programme because it generally falls short of anticipating clinical blood needs. ${ }^{921} 2428$ While FRD can become a potential source of VNRBD, it is a subsistence approach to safeguard against the HBTS blood inventory being depleted. ${ }^{16} 2829$ The WHO recommends a base of regular voluntary blood donors to ensure an adequate, reliable and safe blood supply supported by national policies. ${ }^{4031}$ Yet achieving a blood supply comprised entirely of VNRBD remains a significant challenge in LMIC health systems. ${ }^{2} 92332$ Low rates public participation in VNRBD contribute to ongoing blood shortages. ${ }^{14}$ Not surprisingly, the respondents to our survey highlighted more education of the public and increased health authority involvement in facilitating voluntary participation in VNRBD. This study did not examine the specific patient consequences of delayed blood transfusion or under-transfusion; however, the literature recognises that acute blood loss due to haemorrhage and severe anaemia contributes to preventable mortality in LMICs. ${ }^{53334}$ The frequency of this circumstance is not tracked despite its regularity in LMIC and recommendations by WHO for measurement and benchmarking. ${ }^{27} 303536$

Immunohaematology methods (laboratory testing to ensure compatible blood transfusion and prevent haemolytic reactions) varied by the economic level of the country where the HBTS was located. This suggests that gross national income per capita is a significant factor in available laboratory technology. Methods that use slide or tile agglutination for blood typing are subjective in nature. Further, this technique does not include antibody detection, a crucial part of ensuring blood compatibility between patient and blood donor, particularly for previously transfused patients. ${ }^{37}$ Supply chain and instrumentation may also bear on test methods selection in LMIC. The accessibility of test reagents for blood typing and patient/ donor RBC compatibility testing is one of the critical reasons for the lack of this testing. ${ }^{38}$ Reagent availability and simple testing approaches without strict patient identification can lead to false results and the possibility of the HBTS dispensing incompatible blood. ${ }^{32} 37$ Although this study did not specifically explore the use of RBC antibody panels to identify pretransfusion antibodies in the patient, only two facilities did not routinely perform antibody identification, which may suggest a selection bias for facilities with higher capacity to respond to the survey. For instance, in Uganda, the commercial supplies and reagents needed to perform $\mathrm{RBC}$ antibody detection are not routinely available, leading blood bankers to make homemade reagents. ${ }^{39}$

Evidence-based transfusion practices and PBM principles are relevant in LMIC. ${ }^{40}$ PBM refers to a bundle of evidence-based interventions promoting conservative transfusion practice to optimise medical and surgical patient outcomes. ${ }^{41}$ PBM may help address underlying pathological conditions contributing to anaemia, not due to malignancy, the dominant reason for blood transfusion in our survey. For example, the treatment of anaemia in pregnant women with preoperative haematinics may reduce the need for blood transfusion, prevent unnecessary interventions and conserve the blood supply. ${ }^{43}$ Application of PBM uses multidisciplinary approaches, within and beyond the hospital setting. ${ }^{5} 104142$ In our survey, clinician education was identified as a potential barrier to evidence-based blood transfusion. While more than half of the respondents indicated intermittent educational courses on blood transfusion, the respondents cited a need for regular educational programmes. Considering upstream interventions for maternal health, including primary care providers in educational offerings may facilitate the treatment of anaemia in pregnant women at risk of blood loss. ${ }^{42}$ In many high-income countries, HTCs are required by accrediting bodies. ${ }^{18} 44$ The WHO and international professional societies also promote HTCs to improve patient outcomes related to blood utilisation. ${ }^{2741}$ HTCs were reported as active in most respondent facilities and may serve as a platform to influence clinical transfusion practice, including the adoption of PBM. ${ }^{45}$ HTCs could be influential in addressing blood supply shortfalls, blood wastage, approaches to PBM, and educational needs of practitioners. ${ }^{46}$ Most of the HBTS respondents in this survey have a practising HTC, which suggests that attention to transfusion practice is a priority within these LMIC hospitals. However, as one respondent noted, the HBTS and HTCs are not typically fiscal oversight bodies, so their impact may be blunted.

To our knowledge, this survey is one of the most extensive compilations describing the status of LMIC 
blood transfusion at the hospital level. While previous published data was reported at the national level, this study provided unique insights into HBTS capabilities and challenges in delivering blood transfusion to patients including the use of different approaches to obtain blood products. ${ }^{11} 1424$ Participation was ample and geographically diverse, improved by the availability of the survey in four languages. The purposeful sampling process through known contacts enhanced credibility of the findings by accessing individuals with deep knowledge of the subject matter and context. A common theme cited by the HBTS respondents was the need for ongoing government commitment and support, including the need for financial models, health system funding, and health authority advocacy for VNRBD to assure a reliable blood supply. Beyond the bounds of the HBTS, policy-makers, communities, organisations and individuals can promote the act of blood donation for a resilient healthcare system enabled by a safe and sufficient blood supply. ${ }^{30} 48$

Our study had limitations, including its reliance on email for solicitation of survey participation. The sampling was not an exhaustive compilation of all HBTS in LMICs impacting representativeness, transferability and generalisability to settings beyond those studied. Responses cannot be inferred to represent uniform practices in any given country; we observed variability within countries providing more than a single response. The WHO official languages were narrowed based on knowledge about the invited respondents. The analysis was limited to institutions that transfused blood; input was not solicited from those outside the hospital (eg, Ministries of Health, national blood services or ancillary services) who may also have relevant perspectives. The data collection period overlapped the declaration of the COVID-19 pandemic, which may have influenced blood availability. The questions were not specific to pandemic-related changes, nor did COVID-19 arise in any responses. While gross national income per capita is a standard method of stratification, there may be other explanations, including underlying transfusion-dependent disease burden, the structure of the health system, geographical characteristics or other socioecological factors that impact blood inventory in countries. ${ }^{22}$ Selection, recall and desirability bias may have influenced the responses. No external funding or conflicts of interest were disclosed by the respondents. However, some may have perceived the non-monetary incentive as motivation to participate in this survey.

\section{CONCLUSION}

This comprehensive survey provides insights into the status of hospital-based transfusion service resources and capabilities in LMIC. Availability of blood for transfusion continues to be a dominant problem in hospitals, complicated by factors beyond their direct control. Important findings included the responsibility of the HBTS to generate the facility's blood supply and how inadequate inventories result in the inability to fulfil timely blood transfusion orders. Ironically, delayed blood transfusion or lack of transfusion was rarely captured as an adverse event, despite the implications for poor patient outcomes. This study highlights the gap in tracking the patient-level impact of low blood inventories and insufficient testing resources at the hospital level. HBTSs require materials and supplies to perform high-quality laboratory testing, which may otherwise contribute to adverse patient events. $^{2525}$ We found that pretransfusion testing capabilities vary by the country's economic level, hindering safe transfusion therapy. There is a need for ongoing multidisciplinary education, training on evidence-based transfusion practices and reporting among clinicians. Improvement opportunities may include the engagement of HTCs to increase clinical competencies in blood transfusion, nurture continuing education and promote VNRBD. Based on survey responses, LMIC HBTSs require support from their administrations, communities and governments to improve transfusion safety, including sufficient blood inventories available for timely care of patients who need lifesaving transfusion therapy. These findings highlight areas for further exploration, mainly related to the lack of available blood for transfusions in hospitals and actions to identify and consequently avert adverse transfusion events.

\section{Author affiliations}

${ }^{1}$ Doctor of Public Health Leadership, University of Illinois--Chicago, Chicago, Illinois, USA

${ }^{2}$ American Association of Blood Banks, Bethesda, Maryland, USA

${ }^{3}$ Quality Focus, Moraga, California, USA

${ }^{4}$ Pathology, Johns Hopkins Medicine, Baltimore, Maryland, USA

${ }^{5}$ Laboratory Medicine and Pathology, University of Washington, Seattle, Washington, USA

${ }^{6}$ Department of Pathology, University of Arkansas for Medical Sciences, Little Rock, Arkansas, USA

${ }^{7}$ Pathology, Immunology and Microbiology I Medical Education and Administration, Vanderbilt University Medical Center, Nashville, Tennessee, USA

${ }^{8}$ Hospital Sirio Libanes Blood Bank, Sao Paulo, Brazil

${ }^{9}$ Pathology, Northwell Health, Manhasset, New York, USA

${ }^{10}$ Stanford Health Care, Stanford, California, USA

${ }^{11}$ Pathology and Laboratory Medicine, Children's National Hospital, Washington, District of Columbia, USA

Acknowledgements We appreciate Dr Cyril Jacquot and Dr Maya Makhmudova for assistance with translations. We thank the survey respondents for their time and care completing the survey. We acknowledge the AABB for supporting the Global Transfusion Forum investigators.

Contributors LSB: overall content, conceptualisation, data curation, formal analysis, investigation, methodology, project administration, resources, software, validation, visualisation, writing-original draft (verified the underlying data). JS: conceptualisation, investigation, data curation, writing-review and editing. EB: conceptualisation, investigation, data curation, writing-review and editing. MBP: conceptualisation, investigation, validation, resources, data curation. TSI: conceptualisation, methodology, validation, resources, writing-review and editing. QE: conceptualisation, data curation, resources, writing-review and editing. SW: conceptualisation, data curation. Al: conceptualisation, data curation. WC: conceptualisation, data curation. MD: conceptualisation, data curation, formal analysis, investigation, methodology, resources, project administration, supervision, resources, writing-review and editing (verified the underlying data).

Funding EB is supported by the National Heart Lung and Blood Institute 1K23HL151826-01. 
Competing interests LSB reports personal fees and non-financial support from $X$ CellSystem, GLG, and AABB; LSB is a technical advisor to PAHO. JS is a contractor at Roche Molecular Systems. EB reports personal fees and non-financial support from Terumo BCT, Grifols Diagnostics Solutions and Abbott Laboratories outside of the submitted work; $E B$ is a member of the US Food and Drug Administration (FDA) Blood Products Advisory Committee. TSI is a consultant for Terumo Blood Cell Technologies and Alexion Pharmaceuticals Inc.

Patient consent for publication Not applicable.

Ethics approval Children's National Hospital IRB approved this study as \#Pro00013381. Participants gave informed consent to participate in the study before taking part.

Provenance and peer review Not commissioned; externally peer reviewed.

Data availability statement Data are available on reasonable request. Data are available on reasonable request to the corresponding author.

Supplemental material This content has been supplied by the author(s). It has not been vetted by BMJ Publishing Group Limited (BMJ) and may not have been peer-reviewed. Any opinions or recommendations discussed are solely those of the author(s) and are not endorsed by BMJ. BMJ disclaims all liability and responsibility arising from any reliance placed on the content. Where the content includes any translated material, BMJ does not warrant the accuracy and reliability of the translations (including but not limited to local regulations, clinical guidelines, terminology, drug names and drug dosages), and is not responsible for any error and/or omissions arising from translation and adaptation or otherwise.

Open access This is an open access article distributed in accordance with the Creative Commons Attribution Non Commercial (CC BY-NC 4.0) license, which permits others to distribute, remix, adapt, build upon this work non-commercially, and license their derivative works on different terms, provided the original work is properly cited, appropriate credit is given, any changes made indicated, and the use is non-commercial. See: http://creativecommons.org/licenses/by-nc/4.0/.

\section{ORCID iD}

Linda S Barnes http://orcid.org/0000-0002-5090-0328

\section{REFERENCES}

1 WHO. World health organization model list of essential medicines. Mental and Holistic Health: Some International Perspectives, 2019: 119-34.

2 Roberts DJ, Field S, Delaney M, et al. Problems and approaches for blood transfusion in the developing countries. Hematol Oncol Clin North Am 2016;30:477-95.

3 Roberts N, James S, Delaney M, et al. The global need and availability of blood products: a modelling study. Lancet Haematol 2019;6:e606-e615.

4 World Health Organization (WHO).. Global status report on blood safety and availability 2016. Geneva: World Health Organization, 2017.

5 Bates I, Chapotera GK, McKew S, et al. Maternal mortality in subSaharan Africa: the contribution of ineffective blood transfusion services. BJOG 2008;115:1331-9.

6 Kiguli S, Maitland K, George EC, et al. Anaemia and blood transfusion in African children presenting to hospital with severe febrile illness 2015

7 Bates I, Hassall O, Mapako T. Transfusion research priorities for blood services in sub-Saharan Africa. Br J Haematol 2017;177:855-63.

8 Custer B, Zou S, Glynn SA, et al. Addressing gaps in international blood availability and transfusion safety in low- and middle-income countries: a NHLBI workshop. Transfusion 2018;58:1307-17.

9 Weimer A, Tagny CT, Tapko JB, et al. Blood transfusion safety in subSaharan Africa: a literature review of changes and challenges in the 21st century. Transfusion 2019;59:412-27.

10 Kyeyune-Byabazaire D, Hume HA. Towards a safe and sufficient blood supply in sub-Saharan Africa. ISBT science series, 2018: 104-13.

11 Ifland L. Promoting National blood systems in developing countries. Curr Opin Hematol 2014;21:497-502.

12 Ugwu AO, Madu AJ. Blood transfusion in Sub - Saharan Africa : Historical perspective, clinical drivers of demand and strategies for increasing availability. AJOL 2021;23:14-20.

13 Gallaher JR, Mulima G, Kopp D, et al. Consequences of centralised blood bank policies in sub-Saharan Africa. Lancet Glob Health 2017;5:e131-e132.
14 World Health Organization (WHO). 2016 global status report on blood safety and availability, 2017.

15 van Hulst M, Smit Sibinga CT, Postma MJ. Health economics of blood transfusion safety--focus on sub-Saharan Africa. Biologicals 2010;38:53-8.

16 Barro L, Drew VJ, Poda GG, et al. Blood transfusion in sub-Saharan Africa: understanding the missing gap and responding to present and future challenges. Vox Sang 2018;113:1-11.

17 Kralievits KE, Raykar NP, Greenberg SLM, et al. The global blood supply: a literature review. Lancet 2015;385 Suppl 2:S28.

18 AABB. Fundamental standards for blood banks and transfusion services. 1 edn. Bethesda: AABB, 2018.

19 Waiswa MK, Moses A, Seremba E, et al. Acute transfusion reactions at a national referral hospital in Uganda: a prospective study. Transfusion 2014;54:2804-10.

20 Kanagasabai U, Selenic D, Chevalier MS, et al. Evaluation of the who global database on blood safety. Vox Sang 2021;116:197-206.

21 Mohammed AD, Ntambwe P, Crawford AM. Barriers to effective transfusion practices in Limited-Resource settings: from infrastructure to cultural beliefs. World J Surg 2020;44:2094-9.

22 World Bank. World Bank Country and Lending Groups. [Internet], 2019. Available: https://datahelpdesk.worldbank.org/knowledgebase/ articles/906519-world-bank-country-and-lending-groups

23 Bates I, Manyasi G, Medina Lara A. Reducing replacement donors in sub-Saharan Africa: challenges and affordability. Transfus Med 2007; 17:434-42.

24 WHO Expert Group. Expert consensus statement on achieving self-sufficiency in safe blood and blood products, based on voluntary non-remunerated blood donation (VNRBD). Vox Sang 2012;103:337-42.

25 Bloch EM, Gehrie EA, Ness PM, et al. Blood transfusion safety in Low-Resourced countries: Aspiring to a higher standard. Ann Intern Med 2020;173:482-3.

26 Tancred T, Bates I. Improving blood transfusion services. Best Pract Res Clin Obstet Gynaecol 2019;61:130-42.

27 World Health Organization action framework. Action framework to advance universal access to safe, effective and quality-assured blood products 2020-2023 [Internet], 2020. Available: https://www. who.int/publications/i/item/action-framework-to-advance-uasbloodprods-978-92-4-000038-4

28 Allain J-P, Sarkodie F, Boateng P, et al. A pool of repeat blood donors can be generated with little expense to the blood center in subSaharan Africa. Transfusion 2008;48:735-41.

29 Asenso-Mensah K, Achina G, Appiah R, et al. Can family or replacement blood donors become regular volunteer donors? Transfusion 2014;54:797-804.

30 World Health Organization. AIDE-MEMOIRE for ministries of health developing a national blood system, 2011. Available: http://www. who.int/bloodsafety/publications/am developing_a national_blood system.pdf?ua=1

31 World Health Organization and International Federation of Red Cross and Red Crescent Societies. Towards $100 \%$ voluntary blood donation a global framework for action. 123, 2010.

32 Dzik WS, Kyeyune D, Otekat G, et al. Transfusion medicine in sub-Saharan Africa: conference summary. Transfus Med Rev 2015;29:195-204.

33 Kassebaum NJ, Bertozzi-Villa A, Coggeshall MS, et al. Global, regional, and national levels and causes of maternal mortality during 1990-2013: a systematic analysis for the global burden of disease study 2013. Lancet 2014;384:980-1004.

34 Say L, Chou D, Gemmill A, et al. Global causes of maternal death: a who systematic analysis. Lancet Glob Health 2014;2:e323-33.

35 Thomas J, Ayieko P, Ogero M, et al. Blood transfusion delay and outcome in County hospitals in Kenya. Am J Trop Med Hyg 2017;96:511-7.

36 World Health Organization. Strategic framework for blood safety and availability 2016-2025, 2016.

37 Hume HA. Blood Transfusion in Economically Restricted and Developing Countries. In: Transfusion medicine and hemostasis: clinical and laboratory aspects. 2 edn, 2013: 349-55.

38 Natukunda B, Schonewille $H$, van de Watering $L$, et al. Prevalence and specificities of red blood cell alloantibodies in transfused Ugandans with different diseases. Vox Sang 2010;98:167-71.

39 Natukunda B, Ndeezi G, See Er L, et al. The role of improved pre-transfusion testing in the prevention of delayed serologic transfusion reactions among blood recipients in Uganda: a Randomized Controlled Trial (IPAT Study). ISBT Science Series 2019;14:366-73.

40 Meybohm P, Froessler B, Goodnough LT, et al. "Simplified International Recommendations for the Implementation of Patient Blood Management" (SIR4PBM). Perioper Med 2017;6:5. 
41 Society for the Advancement, Blood Management Inc. SABM administrative and clinical standards for patient blood management programs. 5 edn, 2019.

42 Muñoz M, Stensballe J, Ducloy-Bouthors A-S, et al. Patient blood management in obstetrics: prevention and treatment of postpartum haemorrhage. A NatA consensus statement. Blood Transfus 2019;17:112-36.

43 Daru J, Zamora J, Fernández-Félix BM, et al. Risk of maternal mortality in women with severe anaemia during pregnancy and post partum: a multilevel analysis. Lancet Glob Health 2018;6:e548-54.

44 Duits AJ. Challenges for developing sustainable blood transfusion services in the Caribbean. ISBT Science Series 2013;8.
45 Yazer MH, Lozano M, Fung M, et al. An international survey on the role of the hospital transfusion Committee. Transfusion 2017;57:1280-7.

46 Nahirniak S. Hospital transfusion medicine committees - problems and pitfalls. ISBT Science Series 2017;12:418-21.

47 Opare-Sem O, Bedu-Addo G, Karikari P, et al. Fourteen-Year experience of a tertiary hospital transfusion Committee in West Africa. Transfusion 2014;54:2852-62.

48 WHO Action framework to advance universal access to safe, effective and quality assured blood products [Internet], 2020. Available: https://www.who.int/publications/i/item/action-frameworkto-advance-uas-bloodprods-978-92-4-000038-4 